\title{
Ultraviolet B radiation-induced apoptosis in human keratinocytes: cytosolic activation of procaspase- 8 and the role of Bcl-2
}

\author{
Zerihun Assefa ${ }^{a}$, Marjan Garmyn ${ }^{\mathrm{b}}$, Annelies Vantieghem ${ }^{\mathrm{a}}$, Wim Declercq ${ }^{\mathrm{c}}$, \\ Peter Vandenabeele ${ }^{\mathrm{c}}$, Jackie R. Vandenheede ${ }^{\mathrm{a}}$, Patrizia Agostinis ${ }^{\mathrm{a}, *}$ \\ ${ }^{a}$ Division of Biochemistry, Faculty of Medicine, Catholic University of Leuven, Herestraat 49, B-3000 Leuven, Belgium \\ ${ }^{\mathrm{b}}$ Laboratory of Dermatology, Faculty of Medicine, Catholic University of Leuven, Herestraat 49, B-3000 Leuven, Belgium \\ ${ }^{\mathrm{c}}$ Department of Molecular Biomedical Research, VIB, University of Gent, Ledeganckstraat 35, B-9000 Gent, Belgium
}

Received 8 January 2003; revised 28 February 2003; accepted 4 March 2003

First published online 17 March 2003

Edited by Vladimir Skulachev

\begin{abstract}
In this study, we show that ultraviolet $B$ radiation (UVB)-induced apoptosis of human keratinocytes involves mainly cytosolic signals with mitochondria playing a central role. Overexpression of Bcl-2 inhibited UVB-induced apoptosis by blocking the early generation of reactive oxygen species, mitochondrial cardiolipin degradation and cytochrome $c$ release, without affecting Fas ligand (FasL)-induced cell death. It also prevented the subsequent activation of procaspase-3 and -8 as well as Bid cleavage in UVB-treated cells. Comparative analysis of UVB and FasL death pathways revealed a differential role and mechanism of caspase activation, with the UVB-induced activation of procaspase-8 only being a bystander cytosolic event rather than a major initiator mechanism, as is the case for the FasL-induced cell death. Our results suggest that Bcl-2 overexpression, by preventing reactive oxygen species production, helps indirectly to maintain the integrity of lysosomal membranes, and therefore inhibits the release of cathepsins, which contribute to the cytosolic activation of procaspase-8 in UVB-irradiated keratinocytes.
\end{abstract}

(C) 2003 Federation of European Biochemical Societies. Published by Elsevier Science B.V. All rights reserved.

Key words: Ultraviolet radiation; Apoptosis; Bcl-2; Caspase; Keratinocyte

\section{Introduction}

Chronic exposure to solar ultraviolet (UV) radiation is associated with the onset of various physiological and pathological conditions, including skin cancer. Most of the damages attributed to UV are caused by the UVB (290-320 nm) fraction of sunlight [1]. A hallmark effect of the exposure of human skin to UV radiation in vivo is the formation of sunburn cells representing severely damaged keratinocytes undergoing apoptosis [2]. Since the survival of such DNA-damaged cells increases the risk of malignant transformation, understanding the process of UV-induced apoptosis is crucial for

\footnotetext{
*Corresponding author. Fax: (32)-16-345 995.

E-mail address: patricia.agostinis@med.kuleuven.ac.be (P. Agostinis).

Abbreviations: UV, ultraviolet; FasL, Fas ligand; DISC, deathinducing signaling complex; ROS, reactive oxygen species; NAO, nonyl acridine orange; $\mathrm{AO}$, acridine orange; carboxy- $\mathrm{H}_{2} \mathrm{DCFDA}$, carboxy-2', $7^{\prime}$-dichlorodihydrofluorescin diacetate
}

the development of more effective therapeutic strategies to control the multistep process of skin photocarcinogenesis.

A family of highly specific cysteine proteases known as caspases is responsible for the execution of the apoptotic cell death [3]. Pathways leading to the caspase activation vary with the death-inducing stimulus. The extrinsic pathway of caspase activation occurs following the ligation and trimerization of cell membrane death receptors and the recruitment of adapter molecules like the Fas-associated death domain protein and procaspase- 8 to form the death-inducing signaling complex (DISC), where procaspase- 8 is activated through an autocatalytic cleavage [4]. In the intrinsic pathway, the mitochondria act as integrating sensors of multiple death signals by releasing a number of apoptogenic proteins, such as cytochrome $c$, into the cytosol [5]. Cytosolic cytochrome $c$, together with Apaf-1, leads to the formation of the apoptosome and procaspase- 9 activation [6]. These initiator caspases (caspase- 8 and -9) in turn cleave and activate a group of executioner caspases-3 and -7 orchestrating the final demise of the cell. Proteins of the Bcl-2 family govern the commitment to and progression of apoptosis induced by a variety of stimuli. The pro-survival members of the Bcl-2 family (including Bcl-2 and $\mathrm{Bcl}-\mathrm{X}_{\mathrm{L}}$ ) inhibit the onset or progression of apoptosis by preventing the release of apoptogenic molecules from mitochondria and/or sequestering the pro-apoptotic members of the family like Bid, Bax and Bad [7].

UV-induced apoptosis involves a very complex set of events and a recent study has shown that DNA damage, death receptor activation and reactive oxygen species (ROS) independently contribute to the UV-induced apoptosis [8]. Although some reports have shown that anti-apoptotic members of the Bcl-2 family, which are known to have a limited effect on receptor-driven apoptotic pathways, can protect cells from UVB-induced apoptosis [9-11], none of these studies delineated the molecular events that follow UVB irradiation. In general, data in the literature on the cytotoxic effects of UV irradiation are difficult to reconcile, mainly because of the different UV wavelengths and doses used, as well as the variety of cellular models employed. As a result, the main mechanism of UV-induced apoptosis remains largely elusive.

Therefore, we evaluated these somewhat conflicting results using $\mathrm{HaCaT}$ cells, spontaneously immortalized human keratinocytes [12], and a UVB dose that falls within the physiological range of UV exposure. In particular, we investigated the mechanism of procaspase- 8 activation and the role of the anti-apoptotic protein $\mathrm{Bcl}-2$ in the initiation and progression 
of UVB- as compared to Fas ligand (FasL)-induced apoptosis in this cellular system. Our results show that stable overexpression of Bcl-2 inhibits all apoptotic responses in UVB-irradiated $\mathrm{HaCaT}$ cells, without affecting the FasL-induced responses, suggesting that these two signals trigger divergent pathways of cell death. We could show that in UVB-irradiated cells, procaspase- 8 activation takes place in the cytosol rather than at the DISC and that it is dispensable for the UVB-induced apoptosis. Specific inhibition of lysosomal proteases counteracts the UVB- but not FasL-induced activation of procaspase-8, but does not prevent the induction of cell death. Our results suggest that at physiological doses, UVB induces death receptor-independent apoptotic pathways in which mitochondria play a pivotal role by acting as a sensor and integrator of diverse upstream signals and initiator of downstream execution steps through the release of cytochrome $c$.

\section{Materials and methods}

\subsection{Materials}

All caspase inhibitors and substrates were purchased from Bachem. z-FA-fmk was from Calbiochem while calpeptin and pepstatin A were from Sigma. Antibodies were purchased from Biomol (poly(ADP-ribose) polymerase, PARP), Santa Cruz (caspase-3), Alexis (caspase-8), PharMingen (cytochrome $c$ ) and R\&D Systems (Bid). Soluble Flagtagged FasL was produced in HEK293T cells and purified from the medium. All the inhibitors used in this study were added to culture medium prior to treatment for the period of time indicated in figure legends.

\subsection{Cells, culture conditions and treatments}

HaCaT cells stably overexpressing Bcl-2 were a kind gift from Dr. R. Polakowska (Lille, France). The cells were maintained in Dulbecco's modified Eagle's medium (DMEM, $4.5 \mathrm{~g} / 1$ glucose) supplemented with $10 \%$ fetal calf serum and $1 \mathrm{mg} / \mathrm{ml} \mathrm{G} 418$ (all cell culture media and supplements were purchased from Invitrogen). UVB irradiation was carried out exactly as described before [13]. A dose of $10 \mathrm{~mJ} / \mathrm{cm}^{2}$ UVB was chosen as it falls within the physiological range of UV exposure experienced by human skin and represents approximately three times the minimal erythema dose, equivalent to moderate sunburn assuming a $10 \%$ transmission to the basal layer. To examine the response of the cells to Fas-mediated cell death, FasL was aggregated for $30 \mathrm{~min}$ on ice with anti-Flag antibody before being added to the cells.

\subsection{Preparation of cell extracts}

At the end of the incubation period, both the floating and attached cells were collected and washed once in ice-cold phosphate-buffered saline (PBS). Cells were then lysed exactly as described before [14]. Protein concentrations were estimated with bicinchoninic acid (Pierce).

\subsection{Evaluation of apoptosis and caspase activity}

Cells undergoing apoptosis were viewed and photographed with a Leitz Fluovert light microscope with a $40 \times$ objective. Caspase- 3 activity was assayed exactly as described in [15]. Activation of caspase-8, cytochrome $c$ release and the cleavage of PARP and Bid were analyzed by Western blotting as described before [14].

\subsection{Detection of ROS and cardiolipin level}

Intracellular ROS production was detected by using the ROS-sensitive fluorescent dye 5-(and-6)-carboxy-2',7'-dichlorodihydrofluorescin diacetate (carboxy- $\mathrm{H}_{2}$ DCFDA; Molecular Probes). UVB-treated cells were incubated with carboxy- $\mathrm{H}_{2}$ DCFDA for $30 \mathrm{~min}$ at $37^{\circ} \mathrm{C}$ and then examined using the Leitz Diaplan fluorescence microscope. The level of cardiolipin was analyzed by incubating the irradiated cells in DMEM containing the fluorescent dye nonyl acridine orange (NAO; Molecular Probes) for $30 \mathrm{~min}$ at $37^{\circ} \mathrm{C}$. The fluorescence in 10000 cells per sample was measured by flow cytometry and CellQuest software (FACScan, Becton Dickinson) was used to analyze the data.

\subsection{Lysosomal stability}

The stability of lysosomal membranes was assessed using the acridine orange (AO) relocation method [16]. Cells were preincubated for 15 min with AO and then rinsed twice with PBS before irradiation. After a specified period of incubation in AO-free medium, the relative intensities of red and green fluorescence were simultaneously monitored under a fluorescence microscope using blue light for excitation. AO preferentially localizes in acidic cellular compartments with distinct intracellular distribution from that of rhodamine 123 indicating that it is specifically excluded from mitochondria.

\subsection{Determination of cell proliferation}

Subconfluent cells were irradiated and incubated under normal culture conditions. At each time point after irradiation, the extent of cell survival and proliferation was determined by quantifying the cellular protein content using the naphthol blue black (Acros, Beerse, Belgium) method $[17,18]$.

\section{Results}

\subsection{Bcl-2 overexpression inhibits UVB-induced apoptosis in HaCaT cells by stabilizing important mitochondrial functions}

In this study, we first compared the response of parental (control) $\mathrm{HaCaT}$ cells and $\mathrm{HaCaT}$ cells that stably overexpress $\mathrm{Bcl}-2$ (designated $\mathrm{HaCaT}-\mathrm{Bcl}-2$ cells hereafter) to a UVB dose of $10 \mathrm{~mJ} / \mathrm{cm}^{2}$. Beginning at $8 \mathrm{~h}$ after irradiation, control HaCaT cells showed typical features of apoptotic cells with significant loss of cell volume and widespread membrane blebbing. In contrast, the majority of HaCaT-Bcl-2 cells maintained their normal morphology even $16 \mathrm{~h}$ post irradiation (see below in Fig. 3). A prolonged cell survival and proliferation study indicated that this inhibition was not a temporary delay in cell death, but an effective obliteration of key signaling pathways required for UVB-induced apoptosis (Fig. 1A). Analysis of cytochrome $c$ release from mitochondria showed that unlike in control $\mathrm{HaCaT}$ cells, a significantly diminished level of cytochrome $c$ could be detected in the cytosolic fraction of HaCaT-Bcl-2 cells following UVB irradiation (Fig. 1B). The overexpression of Bcl-2 also inhibited UVB-induced depolarization of the mitochondrial membrane potential which largely occurred about $8 \mathrm{~h}$ after irradiation (data not shown).

Previous studies have implicated ROS in UVB-induced apoptosis [19] and Bcl-2 has been shown to have antioxidant activities in some systems [20,21]. Thus, we analyzed whether UVB-induced oxidative stress was mechanistically involved in the release of cytochrome $c$ and whether the protective effect of Bcl-2 was exerted at this level. In control $\mathrm{HaCaT}$ cells, UVB induced a steady time-dependent increase in ROS production and this was markedly suppressed in HaCaT-Bcl-2 cells (Fig. 1C). Of note, Bcl-2 also prevented the early (1-2 h) induction of ROS, which preceded any detectable cytochrome $c$ release in our system (Fig. 1B). These results are in line with the proposed antioxidant activity of Bcl-2 [20,21] and with the role of ROS as possible early mediators of cellular responses to UVB [19].

Recent studies have suggested that ROS can induce the release of cytochrome $c$ through the peroxidation and eventual degradation of cardiolipin [22,23], an unsaturated phospholipid of the inner mitochondrial membrane which sequesters cytochrome $c$ [22]. We monitored the level of cardiolipin by FACS analysis following UVB irradiation of cells loaded with the fluorescent dye NAO. Fig. 1D shows that UVB irradiation of control $\mathrm{HaCaT}$ cells led to a substantial increase 
A)

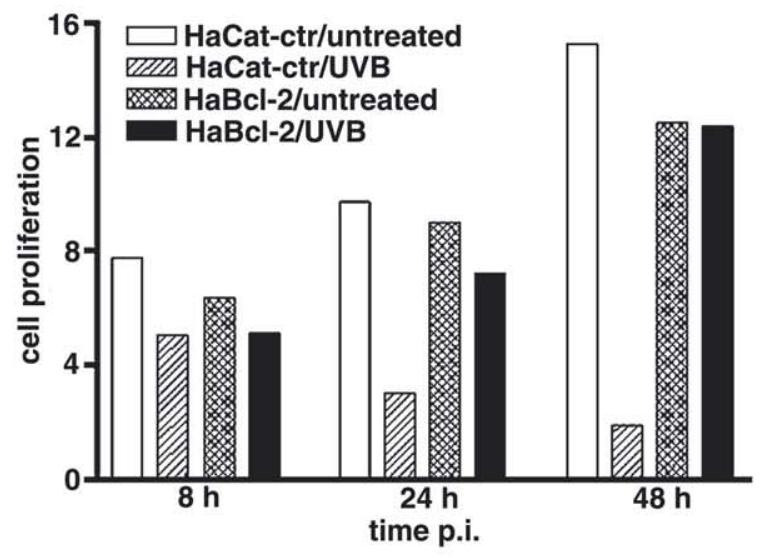

B)

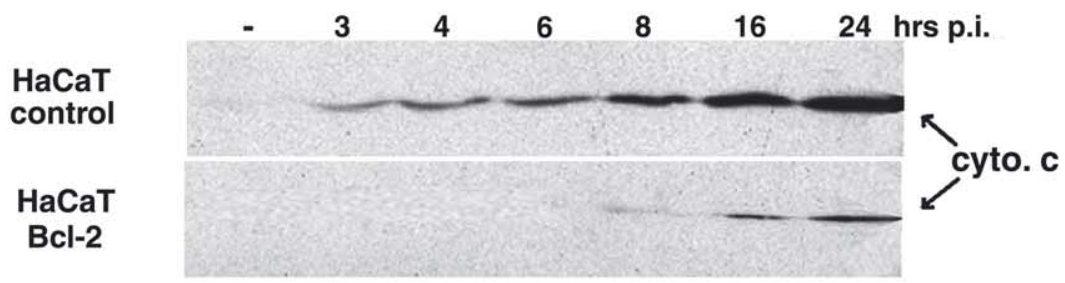

C)
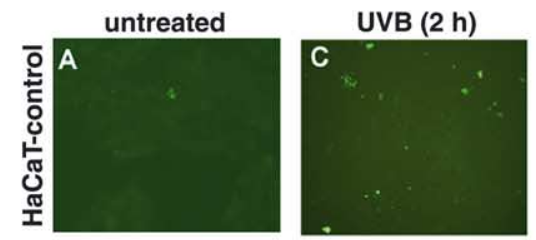

UVB (6 h)
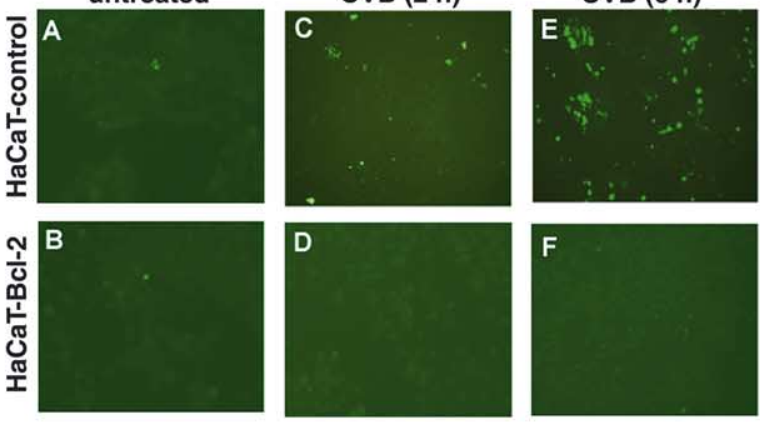

D)
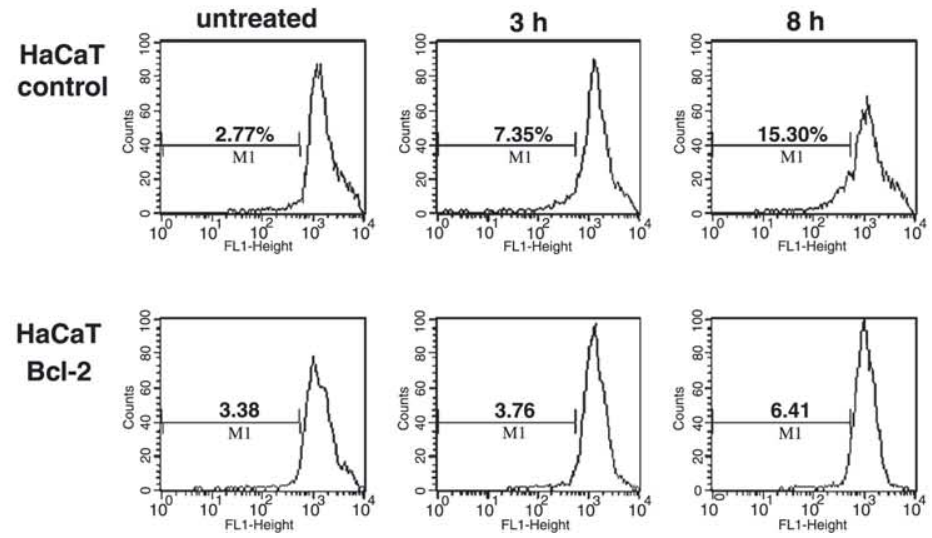

Fig. 1. Bcl-2-overexpressing HaCaT cells are resistant to UVB-induced apoptosis. A: Subconfluent cells were exposed to UVB and the level of cell survival and proliferation was determined at the time points indicated as described in Section 2. A representative of three independent experiments with similar results is shown. B: Cytochrome $c$ redistribution is drastically reduced in Bcl-2-expressing HaCaT cells in response to UVB irradiation. Cells were harvested at the times shown and lysed in digitonin-based buffer. C: Following irradiation, the extent of ROS generation was determined by incubating the cells with $10 \mu \mathrm{M}$ carboxy $-\mathrm{H}_{2}$ DCFDA for $30 \mathrm{~min}$ at $37^{\circ} \mathrm{C}$ and fluorescence microscopy at the indicated time points. Shown are representatives of at least three independent experiments with similar results. D: Control and HaCaT-Bcl-2 cells were exposed to UVB and, at the indicated time points, incubated with $100 \mathrm{nM}$ NAO for 30 min at $37^{\circ} \mathrm{C}$. Cells were then trypsinized and then analyzed for cardiolipin content using flow cytometry. Results from a representative experiment are shown. 
in the percentage of cells with depleted cardiolipin contents whereas in HaCaT-Bcl-2 cells, the extent of cardiolipin degradation was drastically reduced. Altogether, the results suggest a possible mechanism by which $\mathrm{Bcl}-2$ overexpression stabilizes important mitochondrial functions and blocks the release of cytochrome $c$ by increasing the antioxidant capacity of the cells.

\subsection{Bcl-2 inhibits the activation of procaspase-3 and -8 during UVB-induced apoptosis}

In order to further substantiate the protective role of Bcl-2 against UVB-induced apoptosis, we undertook a series of biochemical studies. The activation of procaspase-3, as measured using the fluorogenic substrate Ac-DEVD-amc, was clearly delayed and substantially diminished in HaCaT-Bcl-2 cells (Fig. 2A). Only a modest activity of caspase-3 was detected in HaCaT-Bcl-2 cells, $16 \mathrm{~h}$ after irradiation probably as a result of the small amount of cytochrome $c$ released at this time point. Procaspase- 8 activation involves a two-step processing of the zymogen to generate the mature p18 and p10 subunits through the intermediate $\mathrm{p} 43 / 41$ fragment [24]. As shown in Fig. 2B, the active p18 subunit of caspase- 8 was

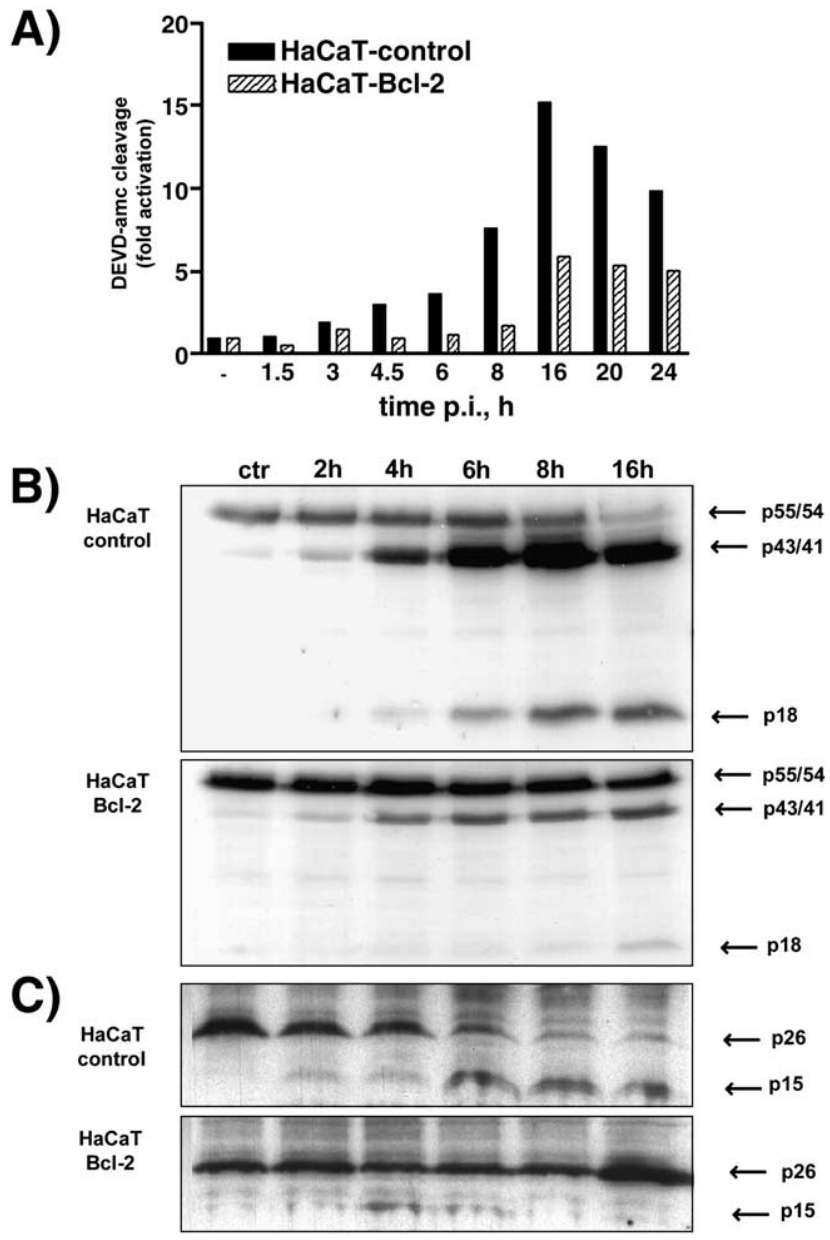

Fig. 2. Biochemical evidence for the resistance of Bcl-2-expressing cells to UVB-induced apoptosis. A-C: UVB-irradiated cells were harvested at the indicated time points after irradiation and assayed for caspase-3 activity (A), procaspase- 8 cleavage (B) and Bid proteolysis (C). A representative result is shown in each case. consistently observed within $4 \mathrm{~h}$ of UVB irradiation in control $\mathrm{HaCaT}$ cells and a complete processing of procaspase- 8 occurred at the 16-h time point, concomitant with the peak of caspase-3 activity (Fig. 2A). In Bcl-2-expressing cells, procaspase- 8 processing was halted after the initial cleavage that generated the p43/41 fragment, and only a very low level of the p18 subunit was detected $16 \mathrm{~h}$ post irradiation (Fig. 2B; see also Fig. 4A). As expected, a time-dependent proteolysis of Bid (loss of the intact protein and/or the appearance of a $15-\mathrm{kDa} \mathrm{tBid}$ ) could be readily observed in UVB-treated control $\mathrm{HaCaT}$ cells and this was almost completely blocked in HaCaT-Bcl-2 cells (Fig. 2C). Together, these results indicate that Bcl-2 inhibits UVB-induced cell death by counteracting apoptotic signals upstream of the activation of caspases.

\subsection{UVB-induced apoptosis involves intrinsic pathways leading to the cytosolic activation of procaspase- 8}

Several studies have suggested an important role for deathreceptor-mediated activation of procaspase- 8 in UV-induced apoptosis [25]. Assuming that UVB-induced apoptosis involves cell surface death receptors as well as cytosolic signals, the results presented above seem to suggest that Bcl-2 overexpression can inhibit both the intrinsic and the extrinsic pathways of cell death. To explore this possibility, control and HaCaT-Bcl-2 cells were treated with UVB, FasL or with a combination of both. Fig. 3 shows that Bcl-2 overexpression clearly blocked the onset of cell death in UVBirradiated but not in FasL-treated cells and that co-treatment with UVB and FasL had additive effects. This differential effect of Bcl-2 on UVB- and FasL-induced apoptosis suggests that the contribution of cell membrane receptors to UVB-induced cell death was negligible. Therefore, it is conceivable that the activation of procaspase-8 in UVB-treated cells occurred in the cytosol. To test this and to identify possible cytosolic activator(s) of procaspase-8 in UVB-irradiated Ha$\mathrm{CaT}$ cells, we employed a range of protease inhibitors.

Cells were incubated for $1 \mathrm{~h}$ prior to irradiation with z-VAD-fmk, a pan-caspase inhibitor that also inhibits other proteases such as cathepsins [26], or with z-FA-fmk (inhibitor of cathepsins B and L [27]). Pretreatment with z-VAD-fmk effectively blocked UVB-induced apoptosis by inhibiting the activation of procaspase-3 and -8 (Fig. 4A,B) and, importantly, the release of mitochondrial cytochrome $c$ (Fig. 4C). On the other hand, more specific caspase inhibitors such as Ac-DEVD-CHO (caspase-3/7), Ac-LEHD-CMK (caspase-9) and Ac-IETD-CHO (caspase-8) had no major effect on cytochrome $c$ release or on the overall UVB-induced cell death (data not shown). In addition, only the cells pretreated with z-VAD-fmk were able to recover and proliferate following UVB irradiation (data not shown), indicating that signals inhibited by either z-VAD-fmk or Bcl-2 overexpression are decisive for the progression of the apoptotic process.

The inhibitor of cathepsins B/L, z-FA-fmk, counteracted the activation of procaspase-8 (Fig. 4A) without affecting either the cleavage of procaspase-3 (Fig. 4B), the release of mitochondrial cytochrome $c$ (Fig. 4C) or the overall cell death (data not shown) in UVB-treated cells. These results not only suggest a novel role for lysosomal proteases in the cytosolic activation of procaspase- 8 but also point out that caspase- 8 activity is not essential for the induction of apoptosis in UVBirradiated cells. 

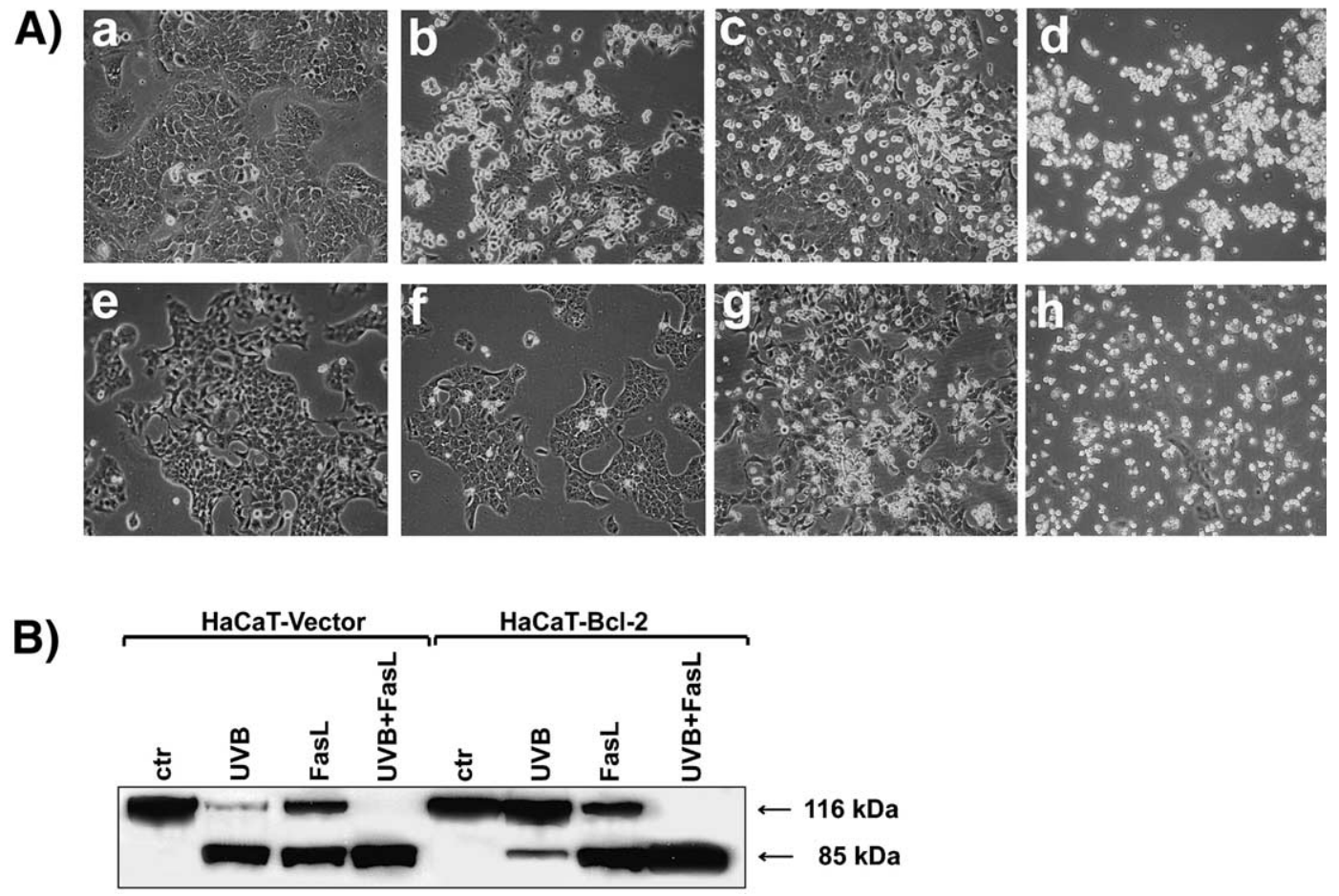

Fig. 3. Bcl-2 inhibits UVB- but not FasL-induced apoptosis. A: Control HaCaT (a-d) and HaCaT-Bcl-2 (e-h) cells were left untreated (a and e), treated with UVB (b and f), FasL (c and g) or a combination of UVB and FasL ( $\mathrm{d}$ and $\mathrm{h}$ ). Light microscopic images were taken $6 \mathrm{~h}$ after treatment. B: Cells were then harvested $8 \mathrm{~h}$ after treatment for the analysis of PARP cleavage.

\subsection{Bcl-2 overexpression maintains the lysosomal membrane integrity in UVB-irradiated cells}

We repeatedly observed that the pattern of inhibition of procaspase- 8 processing by z-FA-fmk was identical to that seen in Bcl-2-overexpressing cells (see Fig. 4A, lanes 4 and $6)$. This could imply that Bcl-2 inhibits the cytosolic processing of procaspase- 8 indirectly by inhibiting either the release or the activities of cathepsins. Thus we tested the possibility that Bcl-2 could stabilize lysosomal membrane integrity [21], by monitoring the lysosomal membrane integrity using the AO uptake/relocation method [16]. Fig. 4D clearly shows that UVB caused a swift deterioration of lysosomal membranes and leakage of their contents into the cytosol, which was inhibited in Bcl-2-overexpressing cells.

Evidence presented so far indicates that the role and the mechanism of activation of caspase- 8 by UVB differ markedly from those of FasL. To corroborate this notion, we compared the effects of the specific cathepsins $\mathrm{B} / \mathrm{L}$ and caspase- 8 inhibitors in control HaCaT cells treated with UVB and FasL. It can be inferred from the PARP cleavage data that in control HaCaT cells, the caspase- 8 inhibitor Ac-IETD-CHO significantly inhibited the FasL death pathway, but had only a marginal or no effect on the UVB-induced apoptosis, while z-FA-fmk did not protect from cell death induced by either UVB or FasL (Fig. 5A). Ac-IETD-CHO significantly reduced both the UVB- and FasL-induced activation of procaspase-8 (Fig. 5B) but had a protective effect only in cells treated with FasL (Fig. 5A and data not shown). Conversely, z-FA-fmk blocked the completion of procaspase- 8 activation in UVBirradiated cells (see also Fig. 4A) but had no effect on its activation during FasL-induced cell death (Fig. 5). Significantly, despite their effect on the activation of procaspase-8, neither Ac-IETD-CHO nor z-FA-fmk was sufficient to block
UVB-induced apoptosis confirming the differential role of this caspase in apoptosis induced by UVB and FasL.

\section{Discussion}

In this report, we provide complementary evidence that a physiologically relevant dose of UVB induces mainly cytosolic signals that lead to mitochondria-driven apoptosis in human keratinocytes. The anti-apoptotic protein $\mathrm{Bcl}-2$ protects more than $90 \%$ of the cells from UV-induced apoptosis by counteracting many, if not all, of the elicited apoptotic signals. Cell proliferation assays confirmed that this inhibition of UVBinduced apoptosis in Bcl-2-expressing cells had long-lasting effects.

Given the multiple signals that are elicited by UVB irradiation, the exact point in the apoptotic signal transduction sequence at which Bcl-2 would act to block cell death is not very clear. In many cellular systems, the anti-apoptotic properties of Bcl-2 are mainly associated with maintenance of normal mitochondrial functions [7]. Recent evidence suggests that Bcl-2 can also guard other organelles partly by fortifying the cellular antioxidant defenses [21]. This was clearly the case in UVB-treated cells, where Bcl-2 overexpression prevented mitochondrial membrane depolarization, formation of ROS, cardiolipin degradation as well as the release of mitochondrial cytochrome $c$. The sequence of events in these UVB-induced changes in mitochondrial functions is not yet clear since they occur within a relatively short span of time. But previous studies have suggested that Bcl-2 can prevent the release of cytochrome $c$ by inhibiting ROS production and the consequent degradation of cardiolipin [28-30]. Our observations indicate that cardiolipin degradation commences at almost the same time as the initial drop in mitochondrial membrane 

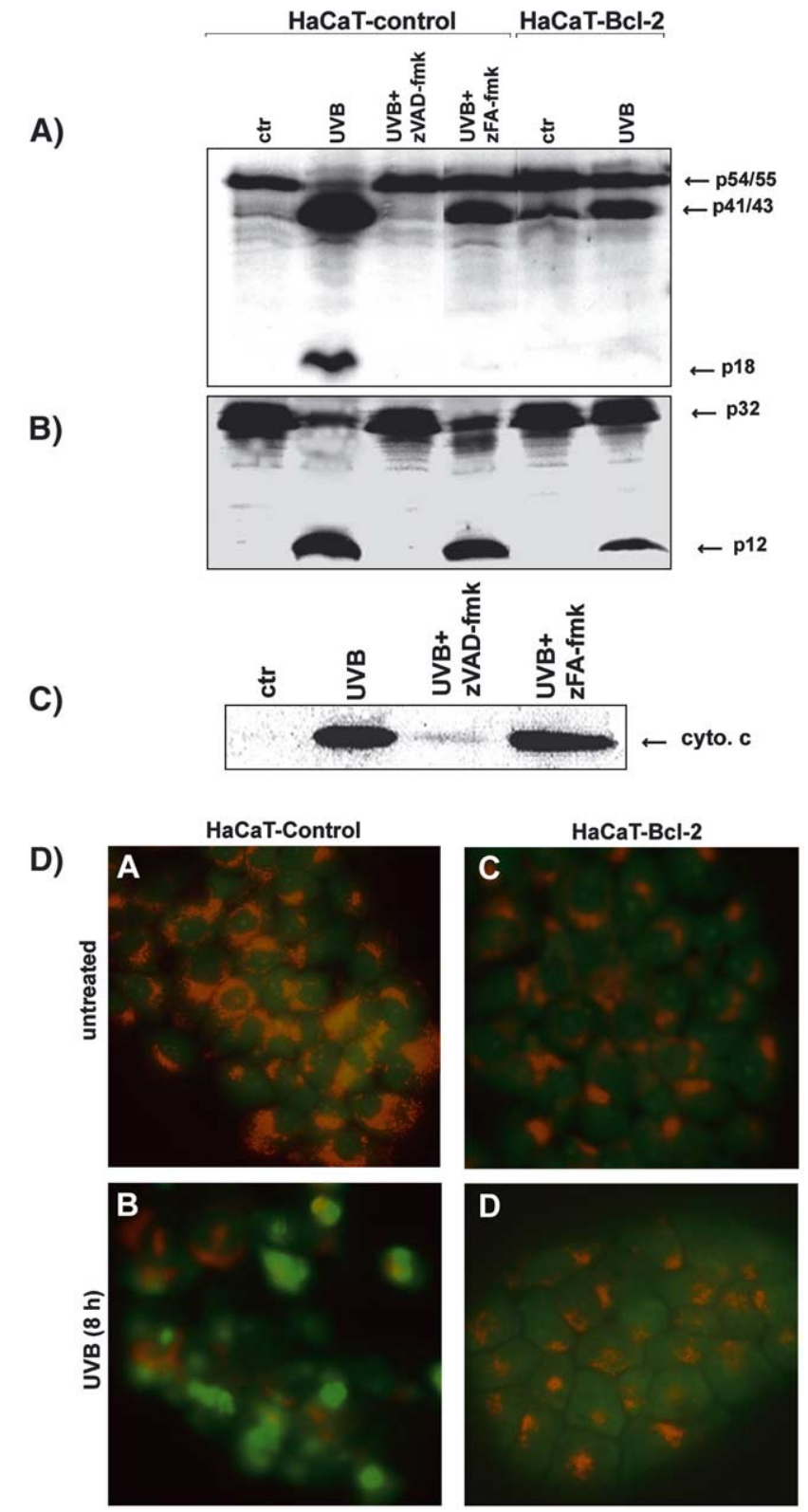

Fig. 4. Effect of protease inhibitors and the response of lysosomes during the UVB-induced apoptosis. Control $\mathrm{HaCaT}$ cells were preincubated with or without z-VAD-fmk $(50 \mu \mathrm{M})$ or z-FA-fmk $(50$ $\mu \mathrm{M})$ for $1 \mathrm{~h}$ before irradiation. The cleavage of procaspase-8 (A) and procaspase-3 (B) was analyzed $16 \mathrm{~h}$ after exposure to UVB by Western blotting. C: The extent of cytochrome $c$ release was assessed $6 \mathrm{~h}$ after irradiation. D: Cells were preincubated for $15 \mathrm{~min}$ with $5 \mu \mathrm{g} / \mathrm{ml} \mathrm{AO}$ and then rinsed twice with PBS before irradiation. Cells were examined $6 \mathrm{~h}$ after irradiation under a fluorescence microscope. A representative of at least three independent experiments is shown.

depolarization but proceeds at relatively faster kinetics (data not shown). Thus, it appears that the time-dependent decrease in NAO signal is not drastically affected by the state of mitochondrial membrane potential. UVB-induced disruption of lysosomal membranes may also be caused by oxidative stress [21], and this may result in the release of a plethora of proteases into the cytosol (see below). Once again, Bcl-2 blocks this oxidative stress-induced disintegration of lysosomal membrane indirectly by inhibiting ROS generation, in line with previous findings [21]. In general, formation of ROS seems to be one of the earliest responses of UVB-irradiated cells as it preceded the release of mitochondrial cytochrome $c$. Overexpression of Bcl-2 blocks ROS generation and precludes the release of cytochrome $c$ and its downstream cascade of apoptotic signals.

Specific inhibition of individual caspases was insufficient to impede UVB-induced cell death. The broad-spectrum protease inhibitor z-VAD-fmk mimicked the effects of Bcl-2 expression in that it also blocked the release of cytochrome $c$, the activation of procaspases- 3 and -8 and restored the proliferative potential of UVB-irradiated cells. The inhibition of procaspase- 8 activation by z-VAD-fmk suggests that cell membrane death receptors were not involved in the initial UVB-induced activation of procaspase- 8 , as the triggering of this pathway would lead to direct autoprocessing and would not be impeded by caspase inhibitors [31]. Thus, the existence of a cleavage and activation mechanism by cytosolic protease(s) could undoubtedly be predicted. Cathepsins B/L, which can be inhibited by z-VAD-fmk [26], would be strong candidates as z-FA-fmk antagonizes the UVB-induced cleavage of procaspase-8, heralding a novel role for these proteases in UVBinduced apoptosis. It is interesting to note that maintenance of lysosomal membrane integrity in UVB-treated HaCaT-Bcl2 cells may be one way through which Bcl-2 blocks procaspase-8 activation. In a clear departure from previously reported observations, our results also indicate that procaspase- 8 activation is not critical for the initiation or completion of UVB-induced apoptosis, but is essentially a bystander effect.

The effect of z-VAD-fmk on the release of cytochrome $c$ might somewhat be surprising in view of previous reports on the caspase independence of this UVB response [14,32]. The significant difference in the dose of UVB used in these studies and the different cell type employed [32] may account for the apparent discrepancy. We have previously shown that a 60 $\mathrm{mJ} / \mathrm{cm}^{2}$ UVB dose induces a p38 mitogen-activated protein

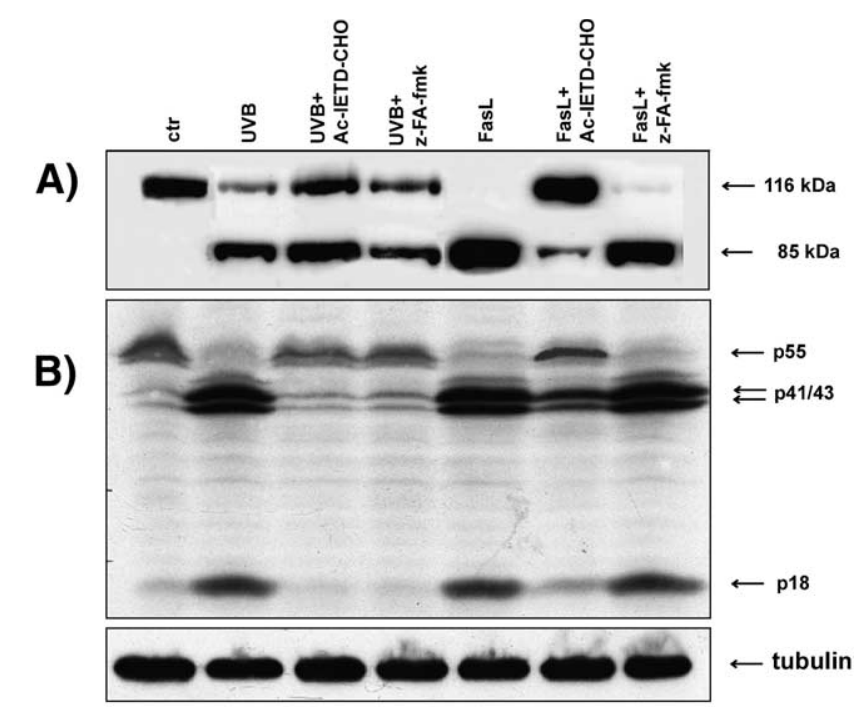

Fig. 5. Differential mechanisms of activation and role of procaspase- 8 in UVB- and FasL-treated cells. Control HaCaT cells were pretreated with $50 \mu \mathrm{M}$ of either Ac-IETD-CHO or z-FA-fmk $1 \mathrm{~h}$ before treatment. Cells were harvested $8 \mathrm{~h}$ later for analysis of the cleavage of PARP (A) and procaspase- 8 (B). The blot was afterwards probed with tubulin monoclonal antibody to ensure loading consistency. 
kinase (MAPK)-mediated, caspase-independent release of mitochondrial cytochrome $c$ in $\mathrm{HaCaT}$ cells [14]. However, the efficiency of PD169316, a specific p38 MAPK inhibitor, in counteracting the release of cytochrome $c$ was dose-dependent, being significantly lower at $10 \mathrm{~mJ} / \mathrm{cm}^{2}$ UVB (unpublished observations). These observations indicate that the relative contribution and the ultimate role of different signals impinging on mitochondria may vary with the dose of UVB.

The inhibition of cytochrome $c$ release by z-VAD-fmk implies a possible involvement of proteases in this mitochondrial response. However, kinetic and inhibitor analyses indicate that neither caspase-3 nor caspase-8 is likely to be involved. Recent studies have convincingly shown that caspase-2 may act upstream of mitochondria to promote cytochrome $c$ release during stress-induced apoptosis [33,34]. These studies suggest that caspase-2, but not caspase- 9 , is an apical caspase in the proteolytic cascade initiated by stress signals. However, it remains to be seen if this also the case in UVB-irradiated cells.

The differential role of Bcl-2 and results from different protease inhibitors indicate that UVB and FasL initiate the process of procaspase-8 activation and cell death in fundamentally different ways. Whereas Bcl-2 has no effect on FasLinduced cell death, a specific caspase-8 inhibitor, Ac-IETD$\mathrm{CHO}$, significantly counteracts the FasL- but not UVB-induced apoptosis. Conversely, z-FA-fmk has no effect on FasL responses while blocking the UVB-induced procaspase8 activation. These results specifically demonstrate distinct mechanisms of procaspase-8 activation and its role during UVB- and FasL-induced cell death in human keratinocytes. Overall, these data again support the notion that UVB irradiation induces mainly the intrinsic pathway of cell death in which cytosolic events, rather than plasma membrane-initiated signals, play a predominant role. Previous evidence for the involvement of cell surface receptors in UVB-induced apoptosis was mostly obtained using either a significantly higher dose of UVB [25] or the more energetic UVC radiation [35]. It is probable that aggregation of the death receptors by UV radiation may require a certain threshold of UV doses. In that case, Bcl-2 would not be expected to interfere and we have also found that the level of protection by Bcl-2 overexpression declines with increased doses of UVB.

It appears that UVB, at doses that fall within the physiological range of exposure of the human skin, initiates apoptotic signals that impinge mainly on mitochondria resulting in cytochrome $c$ release downstream of which all other apoptotic responses are manifested. Since UVB-irradiated cells can only be rescued when the release of cytochrome $c$ is blocked, either by Bcl-2 overexpression or by pretreatment with z-VAD-fmk, we presume that cytochrome $c$ release signifies a point of no return in UVB-induced apoptosis. Scaffidi and colleagues have shown that, with regard to FasL-mediated apoptosis, procaspase-8 activation can occur downstream of mitochondria in type II cells [4]. Bcl-2-overexpressing Jurkat (type II) cells were resistant to FasL-induced apoptosis, with no cytochrome $c$ release or activation of procaspase-8. It is not clear if distinct type I and type II cells exist with respect to UV-induced cell death or if human keratinocytes can be classified as one of the two. However, as the expression of Bcl-2 interferes with UVB-induced apoptotic responses related to mitochondrial cytochrome $c$ release and the activation of the major caspases, we presume that $\mathrm{HaCaT}$ cells behave like type II cells in this context.

Acknowledgements: We thank Dr. R. Polakowska for providing vector and Bcl-2-expressing $\mathrm{HaCaT}$ cells. We gratefully acknowledge the excellent technical assistance of E. Liefooghe and S. Van Kelst. This research was supported in part by Grant P5/12 from Interuniversitaire Attractiepolen (IUAP) of the Federal Belgian Government and by Grant 0211.99 from the Fonds voor Wetenschappelijk OnderzoekVlaanderen.

\section{References}

[1] Madronich, S., McKenzie, R.L., Bjorn, L.O. and Caldwell, M.M. (1998) J. Photochem. Photobiol. 46, 5-19.

[2] Daniels, F., Brophy, D. and Lobitz, W.C. (1961) J. Invest. Dermatol. 37, 351-357.

[3] Hengartner, M.O. (2000) Nature 407, 770-776.

[4] Scaffidi, C., Fulda, S., Srinivasan, A., Friesen, C., Li, F., Tomaselli, K.J., Debatin, K.M., Krammer, P.H. and Peter, M.E. (1998) ЕMBO J. 7, 1675-1687.

[5] Kroemer, G. and Reed, J.C. (2000) Nat. Med. 6, 513-519.

[6] Zou, H., Li, Y., Liu, X. and Wang, X. (1999) J. Biol. Chem. 274, 11549-11556.

[7] Kroemer, G. (1997) Nat. Med. 3, 614-620.

[8] Kulms, D. and Schwarz, T. (2002) Biochem. Pharmacol. 64, 837841.

[9] Takahashi, H., Honma, M., Ishida-Yamamoto, A., Namikawa, K., Miwa, A., Okado, H., Kiyama, H. and Iizuka, H. (2001) Photochem. Photobiol. 74, 579-586.

[10] Haake, A.R. and Polakowska, R.R. (1995) Cell Death Differ. 2, 183-193.

[11] Pena, J.C., Fuchs, E. and Thompson, C.B. (1997) Cell Growth Differ. 8, 619-629.

[12] Boukamp, P., Petrussevska, R.T., Breitkreutz, D., Hornung, J., Markham, A. and Fusenig, N.E. (1988) J. Cell Biol. 106, 761771.

[13] Assefa, Z., Garmyn, M., Bouillon, R., Merlevede, W., Vandenheede, J.R. and Agostinis, P. (1997) J. Invest. Dermatol. 108, 886-891.

[14] Assefa, Z., Vantieghem, A., Garmyn, M., Declercq, W., Vandenabeele, P., Vandenheede, J.R., Bouillon, R., Merlevede, W. and Agostinis, P. (2000) J. Biol. Chem. 275, 21416-21421.

[15] He, J., Whitacre, C.M., Xue, L.Y., Berger, N.A. and Oleinick, N.L. (1998) Cancer Res. 58, 940-946.

[16] Zdolsek, J.M., Olsson, G.M. and Brunk, U.C. (1990) Photochem. Photobiol. 51, 67-76.

[17] Palombella, V.J. and Vilcek, J. (1989) J. Biol. Chem. 264, 18128 18136.

[18] Vantieghem, A., Assefa, Z., Vandenabeele, P., Declercq, W., Courtois, S., Vandenheede, J.R., Merlevede, W., de Witte, P. and Agostinis, P. (1998) FEBS Lett. 440, 19-24.

[19] Pourzand, C. and Tyrrell, R.M. (1999) Photochem. Photobiol. $70,380-390$.

[20] Hockenbery, D.M., Oltvai, Z.N., Yin, X.M., Milliman, C.L. and Korsmeyer, S.J. (1993) Cell 75, 241-251.

[21] Zhao, M., Eaton, J.W. and Brunk, U.T. (2000) FEBS Lett. 485, 104-108.

[22] Shidoji, Y., Hayashi, K., Komura, S., Ohishi, N. and Yagi, K. (1999) Biochem. Biophys. Res. Commun. 264, 343-347.

[23] Ott, M., Robertson, J.D., Gogvadze, V., Zhivotovsky, B. and Orrenius, S. (2002) Proc. Natl. Acad. Sci. USA 99, 1259-1263.

[24] Krueger, A., Schmitz, I., Baumann, S., Krammer, P.H. and Kirchhoff, S. (2001) J. Biol. Chem. 276, 20633-20640.

[25] Leverkus, M., Yaar, M. and Gilchrest, B.A. (1997) Exp. Cell Res. 232, 255-262.

[26] Schotte, P., Declercq, W., Van Huffel, S., Vandenabeele, P. and Beyaert, R. (1999) FEBS Lett. 442, 117-121.

[27] Ahmed, N.K., Martin, L.A., Watts, L.M., Palmer, J., Thornburg, L., Prior, J. and Esser, R.E. (1992) Biochem. Pharmacol. 44, 1201-1207.

[28] Ushmorov, A., Ratter, F., Lehmann, V., Droge, W., Schirrmacher, V. and Umansky, V. (1999) Blood 93, 2342-2352. 
[29] Nomura, K., Imai, H., Koumura, T., Kobayashi, T. and Nakagawa, Y. (2000) Biochem. J. 351, 183-193.

[30] Esposti, M.D., Hatzinisiriou, I., McLennan, H. and Ralph, S. (1999) J. Biol. Chem. 274, 29831-29837.

[31] Yang, X., Chang, H.Y. and Baltimore, D. (1998) Mol. Cell 1, 319-325.

[32] Bossy-Wetzel, E., Newmeyer, D.D. and Green, D.R. (1998) EMBO J. 17, 37-49.
[33] Robertson, J.D., Enoksson, M., Suomela, M., Zhivotovsky, B. and Orrenius, S. (2002) J. Biol. Chem. 277, 29803-29809.

[34] Lassus, P., Opitz-Araya, X. and Lazebnik, Y. (2002) Science 297, $1352-1354$.

[35] Rehemtulla, A., Hamilton, C.A., Chinnaiyan, A.M. and Dixit, V.M. (1997) J. Biol. Chem. 272, 25783-25786. 